\title{
IMPULSIVE SPOT HEATING AND THERMAL EXPLOSION OF INTERSTELLAR GRAINS REVISITED
}

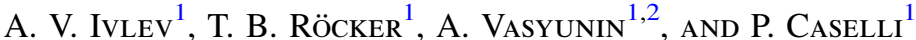 \\ ${ }^{1}$ Max-Planck-Institut für Extraterrestrische Physik, D-85741 Garching, Germany; ivlev@mpe.mpg.de \\ ${ }^{2}$ Ural Federal University, Ekaterinburg, Russia \\ Received 2015 February 16; accepted 2015 March 23; published 2015 May 20
}

\begin{abstract}
The problem of the impulsive heating of dust grains in cold, dense interstellar clouds is revisited theoretically with the aim of better understanding the leading mechanisms of the explosive desorption of icy mantles. We rigorously show that if the heating of a reactive medium occurs within a sufficiently localized spot (e.g., the heating of mantles by cosmic rays (CRs)), then the subsequent thermal evolution is characterized by a single dimensionless number $\lambda$. This number identifies a bifurcation between two distinct regimes: when $\lambda$ exceeds a critical value (threshold), the heat equation exhibits the explosive solution, i.e., the thermal (chemical) explosion is triggered. Otherwise, thermal diffusion causes the deposited heat to spread over the entire grain-this regime is commonly known as whole-grain heating. The theory allows us to find a critical combination of physical parameters that govern the explosion of icy mantles due to impulsive spot heating. In particular, our calculations suggest that heavy CR species (e.g., iron ions) colliding with dust are able to trigger the explosion. Based on recently calculated local CR spectra, we estimate the expected rate of explosive desorption. The efficiency of the desorption, which in principle affects all solid species independent of their binding energy, is shown to be comparable to other CR desorption mechanisms typically considered in the literature. Also, the theory allows us to estimate the maximum abundances of reactive species that may be stored in the mantles, which provides important constraints on the available astrochemical models.
\end{abstract}

Key words: astrochemistry - cosmic rays - dust, extinction - ISM: clouds

\section{INTRODUCTION}

The earliest stages of star formation occur in cold $(T \sim 10$ $\mathrm{K})$, dense $\left(n(H) \gtrsim 10^{4} \mathrm{~cm}^{-3}\right)$, and dark $\left(A_{V} \gtrsim 10 \mathrm{mag}\right)$ molecular cloud cores (e.g., Myers et al. 1987). Under such physical conditions, the rapid freeze-out of molecular species from the gas phase on interstellar grains should occur on a timescale of $\sim 10^{9} / n(H)$ yr. However, while infrared observations confirm the existence of thick icy mantles on interstellar grains (e.g., Gibb et al. 2004), molecular species are also observed in the dark cold gas (Caselli et al. 2002, 2012; Tafalla et al. 2002, 2004). As the lifetime of cold molecular cores is at least $\sim 10^{6} \mathrm{yr}$ (Brünken et al. 2014), a non-thermal desorption mechanism is required to maintain the observed gas-phase abundances of species. The recent discovery of complex organic molecules (Öberg et al. 2010; Bacmann et al. 2012; Cernicharo et al. 2012) and deuterated methanol (Bizzocchi et al. 2014) in the cold gas is further evidence for the nonthermal processing and evaporation of cold icy mantles (Vasyunin \& Herbst 2013b).

The interactions of interstellar grains with cosmic ray (CR) particles, X-ray and UV photons, and even their mutual collisions cause grain heating, and hence stimulate the sublimation of ice (d'Hendecourt et al. 1982; Léger et al. 1985; Hartquist \& Williams 1990; Schutte \& Greenberg 1991; Hasegawa \& Herbst 1993; Shalabiea \& Greenberg 1994; Bringa \& Johnson 2004; Shen et al. 2004; Cuppen et al. 2006; Herbst \& Cuppen 2006; Roberts et al. 2007). Depending on the mechanism of energy deposition, the heated region may be localized or it may extend over the entire grain - these two scenarios are usually referred to as "spot heating" and "whole-grain heating," respectively (Léger et al. 1985; Schutte \& Greenberg 1991; Bringa \& Johnson 2004; Shen et al. 2004). Also, some exothermic reactions occurring on the grain surface (e.g., the formation of molecular hydrogen) may result in local heating and lead to the chemical desorption of weakly bound species (Duley \& Williams 1993; Garrod et al. 2007; Cecchi-Pestellini et al. 2012; Rawlings et al. 2013).

One can identify two distinct regimes of desorption occurring in response to impulsive grain heating: classical thermal evaporation, and the so-called "explosive desorption" triggered by the exothermic chemical reaction(s) between free radicals frozen in the bulk of ice (d'Hendecourt et al. 1982; Léger et al. 1985; Schutte \& Greenberg 1991; Shalabiea \& Greenberg 1994). The essential difference between the two regimes is that the evaporation of the ice mantle (typically limited to the most volatile species) is accompanied by grain cooling, whereas the chemical reactions (activated by the deposited energy) can lead to runaway temperature growth. As a result, explosive desorption may cause the ejection of the entire mantle off the grain surface.

Since the 1980s, there have been various mechanisms proposed to trigger the thermal (chemical) explosion of icy mantles. In particular, these include inelastic collisions between the grains where a certain fraction of their kinetic energy is converted into heat in the mantle (d'Hendecourt et al. 1982; Schutte \& Greenberg 1991; Shalabiea \& Greenberg 1994), and the impact of energetic particles, such as CRs and X-rays (Léger et al. 1985; Shen et al. 2004). The analysis, however, has been almost completely focused on the whole-grain-heating scenario, neglecting the initial thermal spikes emerging in a grain (e.g., along the CR paths). To the best of our knowledge, the possibility of thermal explosion due to CR spot heating was only discussed by Léger et al. (1985), who concluded that such a process is not feasible. ${ }^{3}$

\footnotetext{
3 We note that non-explosive desorption due to spot heating has been studied extensively (e.g., Léger et al. 1985; Hasegawa \& Herbst 1993; Bringa \& Johnson 2004; Shen et al. 2004).
} 
In this article, we revisit the problem of the spot heating of interstellar grains. We introduce the concept of a localized ignition spot and show that the evolution of the initial kinetic energy deposited in a reactive medium in this case is uniquely described by a single dimensionless number $\lambda$. This concept allows us to calculate a critical value of $\lambda$ above which the thermal explosion is triggered, and hence to find the critical combination of physical parameters that govern the explosion of icy mantles due to spot heating. We show that the energy deposited by iron CRs are sufficient to cause such explosions. Furthermore, we demonstrate that the explosion due to wholegrain heating is inhibited by efficient sublimation cooling. Based on the recent calculations of local CR energy spectra, we obtain the minimum expected rate of mantle disruption due to impacts of iron CRs. Finally, the presented theory allows us to estimate the maximum abundances of reactive species that may be stored in the mantles, and thus to impose important constraints on available astrochemical models.

\section{THEORY}

Consider the situation when a certain amount of kinetic energy is "instantaneously" deposited into a reactive medium. It is intuitive to expect that the exact form of the initial energy distribution must be unimportant for its subsequent evolution, provided this distribution is sufficiently localized and the energy is rapidly thermalized. Mathematically, the possibility of a thermal explosion in this case can be investigated by assuming an initial temperature distribution in the form of the delta function. The limits of the applicability of such an approximation of the ignition spot are determined from the numerical analysis, as discussed below.

Let us consider those cases where the initial energy is concentrated on a plane, along an axis, or in a point. For such ignition spots, the problem is characterized by the symmetry indices $D=1,2$, and 3 , respectively, and the heat equation describing the temperature distribution $T(r, t)$ in a reactive medium has the following form (Landau \& Lifshitz 1987):

$$
\rho c \frac{\partial T}{\partial t}=Q_{r} e^{-E_{a} / k_{\mathrm{B}} T}+\kappa\left(\frac{\partial^{2} T}{\partial r^{2}}+\frac{D-1}{r} \frac{\partial T}{\partial r}\right),
$$

with the initial condition

$$
T(r, 0)=\frac{q_{D}}{\rho c} \delta_{D}(r)
$$

Here, $\delta_{D}(r)$ is the delta function in $D$ dimensions, $q_{D}$ is the initial energy density in the ignition spot, $\kappa, \rho$, and $c$ are, respectively, the thermal conductivity, mass density, and specific heat of the medium (treated as incompressible, so $c$ should be taken at constant pressure), $Q_{r}$ is the heat of reaction per unit volume and time, and $E_{a}$ is the relevant activation energy in the Arrhenius factor (definition of $Q_{r}$ and proper choice of $E_{a}$ are discussed in Section 3.1). We first assume the properties of the medium to be independent of the temperature - the cases when $c$ or/and $\kappa$ are functions of $T$ are considered later.

For our analysis of Equations (1) and (2), we normalize the temperature by the activation energy, $\theta=k_{\mathrm{B}} T / E_{a}$. For the dimensionless distance $\xi=r / r_{*}$, we choose the scale $r_{*}$ which provides a unity normalization of Equation (2), while the dimensionless time $\tau=t / t_{*}$ is determined by the timescale $t_{*}$

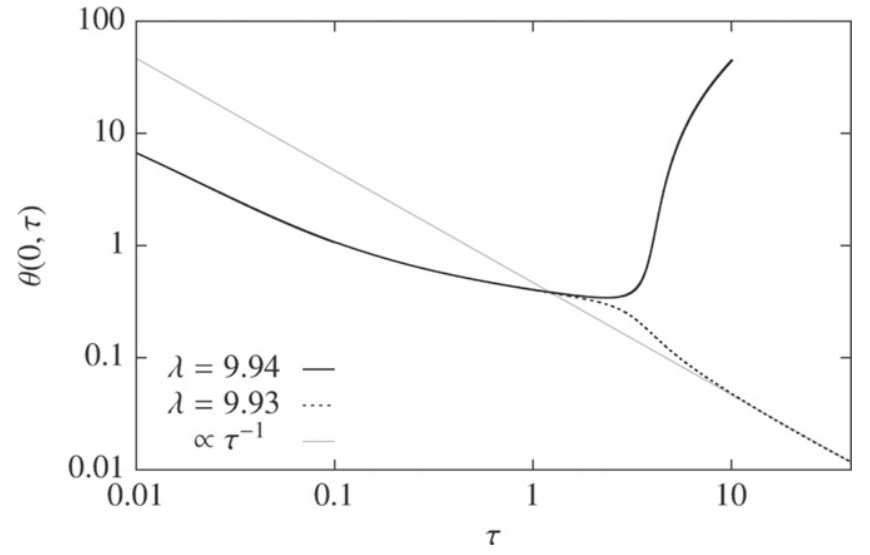

Figure 1. Evolution of the dimensionless temperature at the center of the ignition spot, $\theta(0, \tau)$. Shown are the numerical solutions of Equations (4) and (5) for $D=2$, obtained for marginally under-critical (dashed line) and overcritical (solid line) values of $\lambda$. The under-critical curve tends asymptotically to the solution for $\lambda=0, \theta(0, \tau) \propto \tau^{-D / 2}$ (thin solid line), the over-critical curve approaches $\theta(0, \tau) \propto \tau$ after the explosive growth. For $D=1$ and 3 a similar behavior is observed.

of thermal diffusion at the distance $r_{*}$. This yields

$$
r_{*}=\left(\frac{q_{D}}{\rho c E_{a}}\right)^{1 / D}, \quad t_{*}=\frac{\rho c}{\kappa} r_{*}^{2},
$$

and so the heat equation is reduced to

$$
\frac{\partial \theta}{\partial \tau}=\lambda e^{-1 / \theta}+\frac{\partial^{2} \theta}{\partial \xi^{2}}+\frac{D-1}{\xi} \frac{\partial \theta}{\partial \xi},
$$

and the initial condition to

$$
\theta(\xi, 0)=\delta_{D}(\xi)
$$

Thus, in its dimensionless form, the problem is characterized by a single number:

$$
\lambda=\frac{Q_{r}}{\kappa E_{a}}\left(\frac{q_{D}}{\rho c E_{a}}\right)^{2 / D} .
$$

The role of $\lambda$ is similar to that of the Frank-Kamenetskii number $\lambda_{\mathrm{FK}}$ (see Equation (7)) which governs the thermal stability of a steady state (Frank-Kamenetskii 1969; Landau \& Lifshitz 1987). The relation between $\lambda$ and $\lambda_{\mathrm{FK}}$ is discussed in Appendix A.

The thermal explosion is triggered when $\lambda$ exceeds a certain critical value $\lambda_{\mathrm{cr}}$ - the explosion threshold. From the numerical solution of Equations (4) and (5), we obtain the following thresholds:

$$
\begin{array}{ll}
D=1: & \lambda_{\text {cr }}=1.45 ; \\
D=2: & \lambda_{\text {cr }}=9.94 ; \\
D=3: & \lambda_{\text {cr }}=22.1 .
\end{array}
$$

The bifurcation between the decaying and explosive evolutions is illustrated for $D=2$ in Figure 1, where the temperature at the center of the ignition spot, $\theta(0, \tau)$, is plotted. For $\lambda<\lambda_{\text {cr }}$ the integral effect of thermal diffusion is stronger than that of reaction heating, and so the asymptotic temperature decay is described by the fundamental solution of the heat equation in free space (Landau \& Lifshitz 1987), which yields 


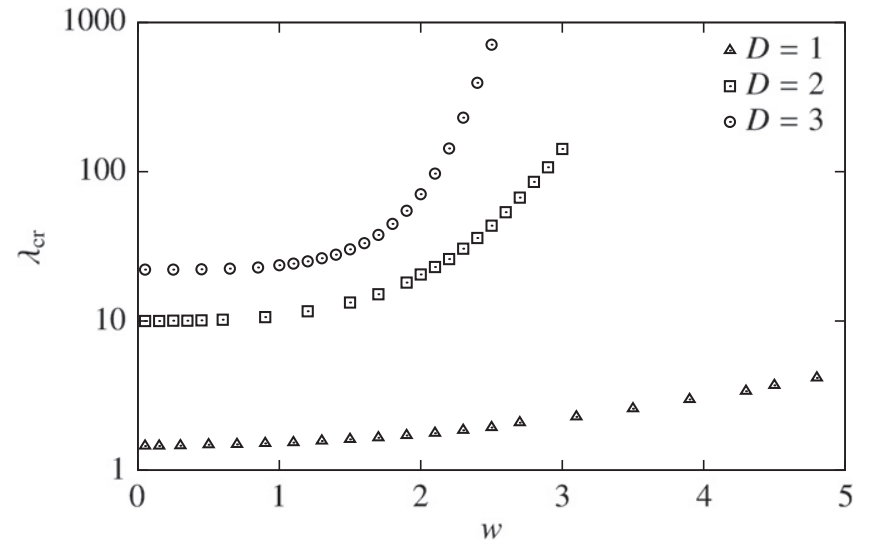

Figure 2. Dependence of the numerically calculated explosion threshold, $\lambda_{\mathrm{cr}}$, on the normalized size of the ignition spot, $w$. The thresholds, plotted for $D=1,2$, and 3, are nearly constant for $w \lesssim 1$, and rapidly increase at larger $w$ (see Appendix A for details). The numerical accuracy for $\lambda_{\text {cr }}$ is better than $\pm 5 \%$.

$\theta(0, \tau) \propto \tau^{-D / 2}$. When $\lambda>\lambda_{\text {cr }}$, thermal diffusion becomes asymptotically negligible and the temperature approaches linear growth, since the Arrhenius term in Equation (4) tends to a constant $(\lambda)$ for large $\theta$. In Figure 1, the bifurcation occurs at $\tau \sim 3$ (while for $D=1$ and 3 it is at $\tau \sim 10$ and $\sim 1$, respectively). We conclude that the explosion develops within a physical time of a few $t_{*}$.

For the numerical solution, we approximate the initial energy distribution by a rectangular function with width $w$ (the delta function formally corresponds to the limit $w \rightarrow 0$ ). The obtained dependence $\lambda_{\mathrm{cr}}(w)$ is plotted in Figure 2, showing that the explosion thresholds remain practically constant for $w \lesssim 1$. Thus, the problem does not (practically) depend on the physical size of the ignition spot as long as it is smaller than $\sim r_{*}$, i.e., the initial energy distribution for such localized spots is well represented by the delta function.

Once the explosion is triggered, the hot reactive zone starts expanding away from the ignition spot. As discussed in Appendix B, the flame front propagates with a constant speed $U$ determined by Equation (8). In Section 3.2, we demonstrate that the magnitude of $U$ is much smaller that the typical sound speed in solids.

The above results can be generalized for the case where the properties of the medium depend on the temperature. In Appendix C, we show that for a power-law temperature dependence of the specific heat, $c(T) \propto T^{\alpha}$ (typical for solids, see Section 3.1), the explosion threshold rapidly decreases with the exponent $\alpha$. Figure 3, illustrating the case $D=2$, demonstrates that for the linear temperature dependence, the value of $\lambda_{\text {cr }}$ decreases by one order of magnitude, and for the quadratic by two. Note that the number $\lambda$, as well as the front speed $U$, in this case are given by Equations (9) and (10). We also demonstrate that the temperature dependence of the thermal diffusivity $\chi=\kappa / \rho c$ has a relatively weak effect on the results.

\section{IMPLICATION FOR INTERSTELLAR DUST GRAINS}

In this section, the theory presented in Section 2 is applied to interstellar dust grains to obtain the conditions when impulsive heating by energetic particles is expected to cause the thermal explosion of icy mantles.

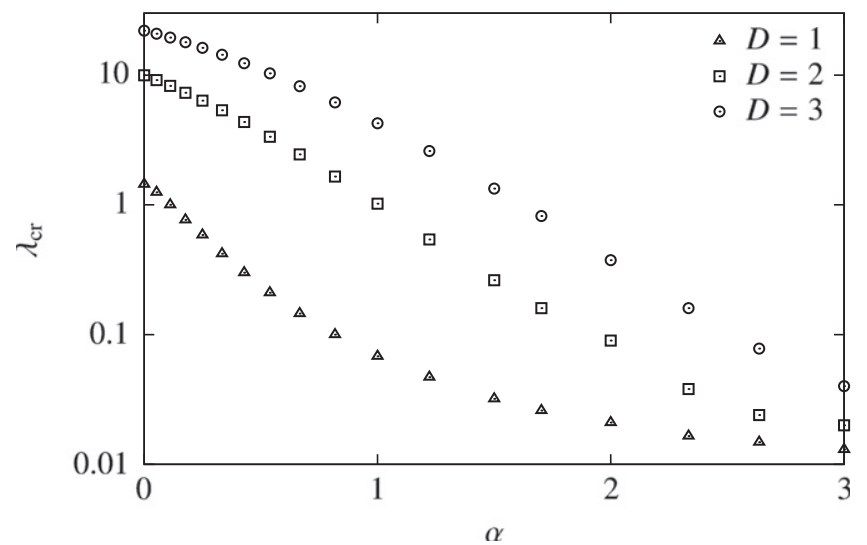

Figure 3. Effect of the temperature-dependent specific heat on the explosion threshold. The critical numbers $\lambda_{\text {cr }}$, numerically calculated for $D=1,2$, and 3 , are plotted vs. the exponent $\alpha$ determining the temperature dependence $c \propto T^{\alpha}$. The numerical accuracy for $\lambda_{\text {cr }}$ is better than $\pm 5 \%$.

The impulsive heating by CR particles, sketched in Figure 4, displays axial symmetry and is described by the solution for $D=2$. The initial energy density $q_{2}$, which enters the dimensionless number $\lambda$ in this case, is equal to the stopping power of a CR particle. The stopping power depends on the particle kinetic energy $\epsilon$ (per nucleon) and exhibits a broad maximum at $\varepsilon=\varepsilon^{\max }$ (Ziegler 1980): for protons, $\varepsilon_{\mathrm{H}}^{\max } \sim 0.1$ $\mathrm{MeV}$ and $q_{2}\left(\varepsilon_{\mathrm{H}}^{\max }\right) \sim 10^{-10} \mathrm{~J} \mathrm{~cm}^{-1}$; for iron ions, $\varepsilon_{\mathrm{Fe}}^{\max } \sim 1$ $\mathrm{MeV} /$ nucleon and $q_{2}\left(\varepsilon_{\mathrm{Fe}}^{\max }\right) \sim 10^{-8} \mathrm{~J} \mathrm{~cm}^{-1}$. The heating by $\mathrm{X}-$ rays is better described by the spherically symmetric solution, $D=3$ (see discussion in Section 3.2).

\subsection{Properties of Icy Mantles}

Let us summarize typical physical properties of mantles which determine the magnitude of $\lambda$.

For many amorphous solids (including ice), the specific heat $c$ increases approximately as $\propto T^{2}$ at lower temperatures, with $10^{-2} \mathrm{~J} \mathrm{~cm}^{-3} \mathrm{~K}^{-1} \lesssim \rho c \lesssim 0.3 \mathrm{~J} \mathrm{~cm}^{-3} \mathrm{~K}^{-1}$ for $10 \mathrm{~K} \leqslant T \leqslant 50 \mathrm{~K}$; the growth becomes slower at higher temperatures, $\rho c \sim 3$ $\mathrm{J} \mathrm{cm}^{-3} \mathrm{~K}^{-1}$ at $T \sim 10^{3} \mathrm{~K}$ (Zeller \& Pohl 1971; Léger et al. 1985). We employ this generic dependence for the estimates below. For thermal conductivity $\kappa=\rho c \chi$, we use the diffusivity $\chi \sim 10^{-2} \mathrm{~cm}^{2} \mathrm{~s}^{-1}$ (d'Hendecourt et al. 1982; Léger et al. 1985; Schutte \& Greenberg 1991); the latter is approximately constant for many amorphous solids at $T \gtrsim 30$ K (Zeller \& Pohl 1971). Note that $\chi$ may decrease with $T$ for amorphous water ice (Andersson \& Suga 2002), but this should only have a minor effect on the results (see Appendix C).

For the sake of clarity, we suppose that among the variety of reactive species (radicals) stored in the mantle, there is a pair $(A$ and $B$ ) whose exothermic reaction dominates the heat release (the approach can be straightforwardly generalized to multiple reactions). The heat rate is then given by (Léger et al. 1985) $Q_{r} \simeq E_{r} \varphi_{A} \varphi_{B} N \nu$, where $E_{r} \sim 3 \mathrm{eV}$ is the typical energy release per reaction, $\varphi_{A, B}=N_{A, B} / N$ are the fractional abundances of the species, $\nu \simeq 2 \times 10^{12} \mathrm{~s}^{-1}$ is their characteristic vibration frequency, and $N \simeq 3 \times 10^{22} \mathrm{~cm}^{-3}$ is the total number density of molecules in the ice (Léger et al. 1985; Schutte \& Greenberg 1991). Thus, to estimate the magnitude of 

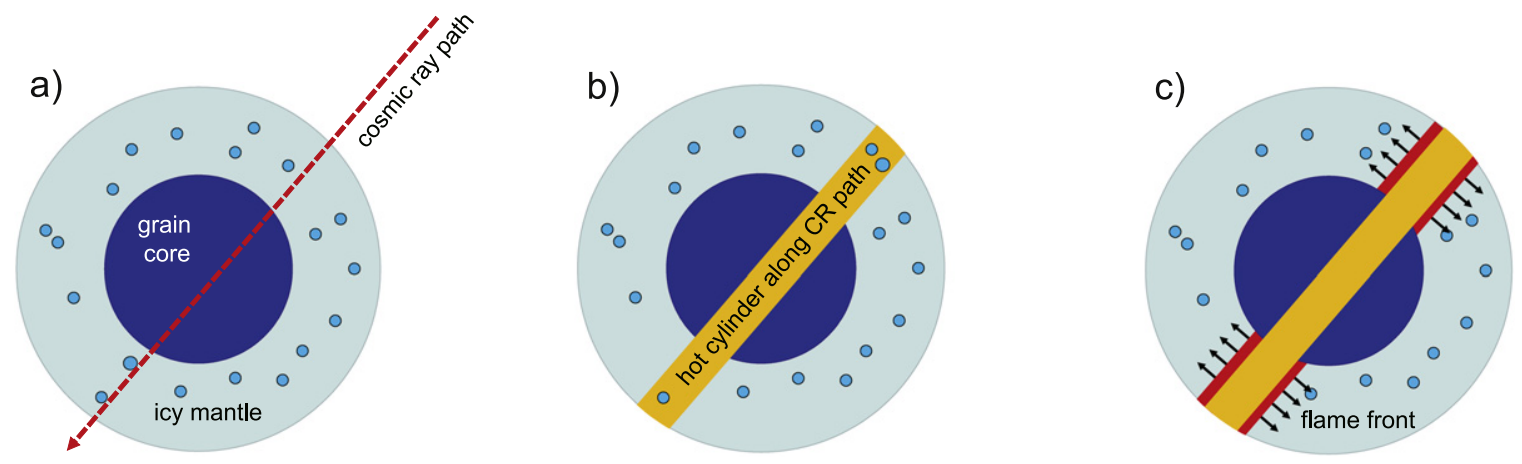

Figure 4. Schematic representation of the thermal (chemical) explosion of an icy mantle due to a cosmic ray impact. (a) A refractory core of a grain is covered by a thick icy mantle where some reactive species (radicals, blue dots) are stored in bulk. Each collision of a CR particle with a grain is accompanied by a loss of energy, deposited along the CR path. (b) This creates a hot, narrow cylindrical region whose subsequent evolution is governed by the dimensionless number $\lambda$, given by Equation (6): if $\lambda$ is below a certain critical value, the deposited energy is simply redistributed over the grain's volume (the whole-grain-heating scenario). Otherwise, the thermal explosion is triggered (c) and runaway exothermic reactions generate a cylindrical flame front in the mantle, leading to its disruption.

$Q_{r}$, we need to know the abundance of the reactive species. Let us elaborate on this point.

Direct infrared observations of interstellar ices can only supply us with the abundances of major ice constituents, which are in general not reactive under cold ISM conditions, with the exception of $\mathrm{CO}$ ice. As such, we have to rely on astrochemical modeling when estimating the abundances of reactive species in typical interstellar ice. Early astrochemical models did not have make distinction between the reactive surface and the more inert bulk of a thick icy mantle (Hasegawa et al. 1992; Hasegawa \& Herbst 1993). Therefore, in these models, all of the species adsorbed on a grain surface participate in efficient "surface" chemistry and the resulting fraction of radicals stored in the mantle is very low. However, in a number of more recent studies, several important effects were recognized which favor larger amounts of radicals being stored in the interstellar ice. First, icy mantles in dark clouds are likely to be thick and to consist of several hundreds of monolayers (see, e.g., Section 4.2 of Caselli \& Ceccarelli 2012). For this reason, reactive species in the inner layers of the ice may be quickly covered by new accreting species during ice formation and become excluded from the rapid surface chemistry. Reactive species become frozen into water ice and thus survive and accumulate (e.g., Taquet et al. 2012). Moreover, it is likely that icy mantles are exposed to UV photons even in dark clouds. Photons can penetrate the entire mantle and dissociate stable molecules in the ice (Chang \& Herbst 2014; Cruz-Diaz et al. 2014), thus producing radicals (Garrod 2013a). Finally, the amount of radicals in the ice may be affected by the internal ice structure: theoretical studies show that the porous structure of ice favors the accumulation of radicals (Taquet et al. 2012). However, some authors show that interstellar ices are rather more compact than porous (Garrod 2013b).

To obtain quantitative estimates of the fraction of reactive species stored in the ice in a dark cloud, we simulate the formation of the icy mantle during the contraction of a diffuse cloud into a dense core using our MONACO code and a simple evolutionary model presented in Vasyunin \& Herbst (2013a). Briefly, in the evolutionary model, the temperature linearly decreases with time from 20 to $10 \mathrm{~K}$, and the gas density increases from $10^{3} \mathrm{~cm}^{-3}$ in the beginning to $10^{5} \mathrm{~cm}^{-3}$ at the end of the contraction. Visual extinction $A_{V}$ increases selfconsistently with density from $A_{V}=3$ to $A_{V} \geqslant 10$. The MONACO code has been updated in comparison to Vasyunin \& Herbst (2013a), and it now includes chemistry in the bulk

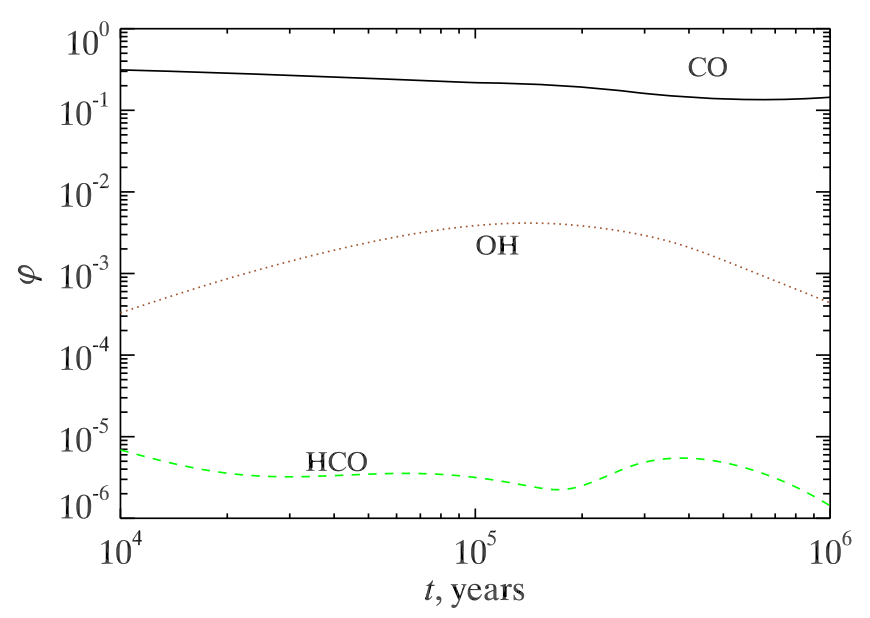

Figure 5. Evolution of the fractional abundances of the major reactive species (with respect to the total number of molecules in the ice). Results are from the numerical modeling with the modified MONACO code.

due to ice photoprocessing and the intramantle diffusion of species (details will be described in a future paper).

The mobility of species in the bulk of the ice is likely to be significantly lower than on the surface, due to the larger number of neighboring species that bonded to each other (Garrod 2013a). In our microscopic formalism, this means a higher diffusion energy for a species in the bulk than on the surface. Following Garrod (2013a), we set the diffusion energy of the species in the bulk to be two times the respective surface diffusion energy. The latter, in turn, is usually taken as a fraction of the sublimation enthalpy (typically, their ratio varies from 0.3 to 0.8 , see, e.g., Hasegawa et al. 1992; Ruffle \& Herbst 2000); here, we chose a value of 0.5 in agreement with the best-fit model by Vasyunin \& Herbst (2013a). As such, the diffusion energy is $\simeq 1150 \mathrm{~K}$ for $\mathrm{CO}$ molecules, and so $\mathrm{CO}$ as well as other abundant reactive species (with higher diffusion energies) produced during ice photoprocessing and entrapment of accreting material can effectively accumulate in the bulk of ice. Thus, we will consider the CO diffusion energy as the relevant activation energy for the Arrhenius factor in Equation (1), i.e., $E_{a} / k_{\mathrm{B}}=1150 \mathrm{~K}$.

In Figure 5, the fractional abundances $\phi$ of the most abundant reactive species in the ice are plotted versus time. CO is mainly accreted from the gas phase. Some of it undergoes 
hydrogenation and ultimately converts to methanol and other saturated species, but a significant fraction of $\mathrm{CO}$ molecules become buried in the icy mantle in a pristine form. The next most abundant species is $\mathrm{OH}$, which is mainly produced via the dissociation of water by photons and CR protons (according to Andersson \& van Dishoeck 2008, only a fraction of the dissociation products recombine back to $\mathrm{H}_{2} \mathrm{O}$ ). Note that the abundance of $\mathrm{OH}$ in our model is about two orders of magnitude lower than in other models of multilayer ice (e.g., Taquet et al. 2012). Presumably, this is due to the fact that we take into account the efficient recombination of $\mathrm{OH}$ with free $\mathrm{H}$ atoms that are generated in the bulk of ice and perform a random walk before reaching the ice surface. Finally, a certain fraction of $\mathrm{HCO}$ is produced in the bulk, mainly in dissociation of methanol by $\mathrm{CR}$ protons. We see that the abundances of $\mathrm{CO}$ and $\mathrm{OH}$ reach the values of $\varphi_{\mathrm{CO}} \sim 10^{-1}$ and $\varphi_{\mathrm{OH}} \sim 3 \times 10^{-3}$ at later stages of the contraction. We employ these characteristic values for the estimates below.

\subsection{Explosion Due to Spot Heating}

In Section 2, we pointed out that the presented theory can be used as long as the physical size of the ignition spot does not exceed $\sim r_{*}$. By substituting typical parameters (listed above, with $\rho c=0.3 \mathrm{~J} \mathrm{~cm}^{-3} \mathrm{~K}^{-1}$ ) in Equation (3) for $D=2$, we obtain $r_{*} \sim 3 \times 10^{-6} \mathrm{~cm}$ for heating by iron CRs. This value is substantially larger than the diameter of the cylindrical volume where CRs deposit their energy $(\lesssim 100 \AA$ A , Léger et al. 1985) and, at the same time, is smaller than the size of the large grains dominating the interstellar dust mass $\left(\sim 10^{-5} \mathrm{~cm}\right)$. Furthermore, the cylindrical explosion develops during a time of the order of $3 t_{*} \sim 3 \times 10^{-9} \mathrm{~s}$, which is much longer than the time during which the deposited CR energy is thermalized $\left(\lesssim 10^{-11} \mathrm{~s}\right.$, Léger et al. 1985). Thus, the theory is indeed applicable to study the reaction of large grains on impulsive heating by heavy CR species (assuming dust properties that are typically used in astrochemical modeling, see Section 3.1).

Let us estimate the magnitude of $\lambda$ for individual collisions with iron CRs. First, we assume a constant $c$ (and $\kappa$ ) for icy mantles. By substituting into Equation (6) $q_{2} \sim 10^{-8} \mathrm{~J} \mathrm{~cm}^{-1}$ and $\rho c=0.3 \mathrm{~J} \mathrm{~cm}^{-3} \mathrm{~K}^{-1}$, and setting $E_{r}=3 \mathrm{eV}$ and $\varphi_{\mathrm{CO}} \varphi_{\mathrm{OH}}=3 \times 10^{-4}$ for the reaction between $\mathrm{CO}$ and $\mathrm{OH}$, we obtain $\lambda \sim 30$, which exceeds $\lambda_{\text {cr }} \simeq 10$ for $D=2$. Hence, iron ions with energy corresponding to the maximum of the stopping power are able to trigger the explosion, ${ }^{4}$ whereas CR protons with $q_{2} \sim 10^{-10} \mathrm{~J} \mathrm{~cm}^{-1}$ remain under-critical, since $\lambda \propto q_{2}$.

Remarkably, when the temperature dependence of the specific heat is taken into account, the resulting ratio $\lambda / \lambda_{\text {cr }}$ becomes even larger, i.e., the explosion condition is relaxed in comparison with the constant- $c$ case. For $c(T) \propto T^{\alpha}$, we employ the results of Appendix $\mathrm{C}$ and calculate the enthalpy scale $H_{E}$ with $\rho c(E) \sim 3 \mathrm{~J} \mathrm{~cm}^{-3} \mathrm{~K}^{-1}$ and $1 \leqslant \alpha \leqslant 2$; using the dependence $\lambda_{\mathrm{cr}}(\alpha)$ plotted in Figure 3 , and substituting $H_{E}$ into Equation (9), we obtain $\lambda / \lambda_{\text {cr }}$ in the range between $\sim 3$ and $\sim 30$ for iron CRs. Thus, even if some of the numbers used above for

\footnotetext{
4 The explosion threshold could also be evaluated by directly comparing the rates of chemical reaction and thermal diffusion in Equation (1). However, as shown in Appendix A, this comparison should be performed at the "optimum moment" (shortly before the bifurcation in Figure 1). Presumably, this latter point was not taken into account by Léger et al. (1985), who concluded that thermal explosion due to spot heating is unlikely.
}

estimating $\lambda$ were somewhat less favorable (e.g., if $\varphi_{A} \varphi_{B} \sim 3 \times 10^{-5}$ ), iron CRs should still lead to the explosion.

The flame front, generated in the mantle by the explosion, propagates with the speed $U$ given by Equations (8) or (10). From this, we obtain $U \sim 10^{4} \mathrm{~cm} \mathrm{~s}^{-1}$, which is more than an order of magnitude lower than the typical sound speed in ice (see, e.g., Vogt et al. 2008). The crossing time in a grain with radius $a$ is $\sim a / U \sim 10^{-9} \mathrm{~s}$ for $a \sim 10^{-5} \mathrm{~cm}$, and so one would expect a practically instant mantle evaporation. However, one should note that the flame front exerts enormous stress-the thermal pressure $\sim N T$ substantially exceeds the GPa level, while the tensile strength of ice is less than one MPa (e.g., Petrovic 2003). This may lead to the mechanical disruption of the mantle before it completely evaporates.

One can estimate the rate of mantle disruption due to thermal explosions, $1 / t_{\text {dis }}$, which is determined by the local energy spectrum of iron CRs. We assume a constant abundance of iron ions of $\phi_{\mathrm{Fe}} \sim 10^{-4}$ (relative to protons, see, e.g., Léger et al. 1985; Shen et al. 2004) and employ the local proton spectrum $J_{\mathrm{H}}(\varepsilon)$ from Padovani et al. (2009), where $\epsilon$ is the energy per nucleon. The disruption rate is equal to the product of the grain cross section and the CR flux contributing to the explosion. The minimum value of the latter can be roughly estimated as $^{5} \sim 4 \pi \phi_{\mathrm{Fe}} \varepsilon_{\mathrm{Fe}}^{\max } J_{\mathrm{H}}\left(\varepsilon_{\mathrm{Fe}}^{\max }\right)$, where $\varepsilon_{\mathrm{Fe}}^{\max } \sim 1 \mathrm{MeV} /$ nucleon corresponds to the maximum stopping power for iron ions (Ziegler 1980). Even for dense clouds (with a column density for molecular hydrogen of $\sim 3 \times 10^{22} \mathrm{~cm}^{-3}$, where the spectrum is strongly attenuated; Padovani et al. 2009), we determine that the disruption rate for large grains $\left(a \sim 10^{-5}\right.$ $\mathrm{cm})$ is not lower than

$$
1 / t_{\mathrm{dis}} \sim(2 \pi a)^{2} \phi_{\mathrm{Fe}} \varepsilon_{\mathrm{Fe}}^{\max } J_{\mathrm{H}}\left(\varepsilon_{\mathrm{Fe}}^{\max }\right) \sim 10^{-6} \mathrm{yr}^{-1} .
$$

Furthermore, supposing that the entire mantle evaporated upon disruption, we can also estimate the minimum desorption rate of molecules into the gas phase. For a mantle with thickness $\Delta a$, the desorption rate of species $A$ is $\sim 4 \pi a^{2} \Delta a N \varphi_{A} / t_{\text {dis }} \propto a^{5}$ (assuming $\Delta a \propto a$ ). We see that the explosive desorption is heavily dominated by large grains with a desorption rate of the order of $3 \times 10^{-7}$ molecules grain ${ }^{-1} \mathrm{~s}^{-1}$ for $\mathrm{CO}$ molecules. This value is comparable to the desorption rates due to a combination of other mechanisms (Shen et al. 2004; Herbst \& Cuppen 2006), such as explosion due to whole-grain heating (see next section for its critical discussion), and evaporation due to whole-grain and spot heating. We note that the calculations of the desorption rate reported earlier (Léger et al. 1985; Hartquist \& Williams 1990; Hasegawa \& Herbst 1993; Bringa \& Johnson 2004; Shen et al. 2004; Herbst \& Cuppen 2006) do not take into account the attenuation of the local CR spectrum, which is included in our analysis.

It is noteworthy that we can practically exclude other sorts of energetic particles (e.g., X-rays or UV photons) as possible causes of explosion due to spot heating. To demonstrate this, let us consider X-rays as the most energetic species among such particles. The maximum energy which can be deposited in a

\footnotetext{
To obtain the CR flux contributing to the explosion, one should integrate $J_{\mathrm{H}}(\varepsilon)$ over the range of energies (around $\varepsilon_{\mathrm{Fe}}^{\max }$ ) where $q_{2}(\varepsilon)$ exceeds the critical value, i.e., where $\lambda\left(q_{2}\right) \gtrsim 10$. Given the uncertainties in the local spectrum in this range (Padovani et al. 2009), only the lower bound of the flux can be reasonably estimated.
} 
grain by an X-ray photon is limited by the condition that the stopping range of electrons produced by the photon is smaller than the grain size; for $a \sim 10^{-5} \mathrm{~cm}$, we get an upper energy limit of the order of a few $\mathrm{keV}$ (see, e.g., Léger et al. 1985). Since energetic electrons lose most of the energy at the end of their paths, the spherically symmetric solution is more appropriate to describe the problem in this case. For $D=3$ (and otherwise the same parameters as above), from Equation (6) we determine that the minimum ignition energy to satisfy the condition $\lambda>\lambda_{\text {cr }} \simeq 22$ is $q_{3} \sim 10^{5} \mathrm{eV}$, which exceeds the maximum deposited energy by about two orders of magnitude.

Finally, the presented theory allows us to impose important constraints on the fractional abundance of reactive species in icy mantles, and thus to discriminate between different astrochemical models. In particular, one can estimate the upper limit of the abundance of radicals which can be stored in a mantle: for example, some models predict that at later stages of the cloud evolution, the product of the relative abundances of such radicals may be as high as $\varphi_{A} \varphi_{B} \sim 3 \times 10^{-3}$ (or even higher, see, e.g., Schutte \& Greenberg 1991; Shalabiea \& Greenberg 1994; Taquet et al. 2012; Chang \& Herbst 2014). Since $\lambda \propto q_{2} \varphi_{A} \varphi_{B}$, its value for iron CRs would then be about two orders of magnitude larger than $\lambda_{\mathrm{cr}}$, and so the obtained abundances could already be marginally sufficient to satisfy the explosion condition for $\mathrm{CR}$ protons. However, the latter are $\sim 10^{4}$ more abundant than iron CRs, and so the very possibility of mantle explosion due to impacts of CR protons would imply unrealistically high disruption rates of $\sim 10^{-3} \mathrm{yr}^{-1}$ or even larger (these exceed the freeze-out rates at typical molecular cloud densities, i.e., icy mantles simply would not have time to grow). Hence, such high abundances of radicals can be ruled out based on the explosion theory.

\subsection{On the Whole-grain Heating}

If the stopping power of an energetic particle colliding with a grain is too low (under-critical), the deposited energy is rapidly redistributed over the whole grain. Even though the overall temperature increase in this case could be only a few tens of degrees, this leads to an exponential amplification of the chemical heating in the entire reactive volume of the grain, with important consequences for surface chemistry and the chemical composition of icy mantles. The thermal stability in this regime is determined by the global balance between volume heating and surface cooling due to thermal radiation and sublimation (Léger et al. 1985; Schutte \& Greenberg 1991; Cuppen et al. 2006). Therefore, it has usually been argued that there is a critical temperature for whole-grain heating, above which the explosion must be triggered (d'Hendecourt et al. 1982; Léger et al. 1985; Schutte \& Greenberg 1991; Shalabiea \& Greenberg 1994; Shen et al. 2004). As we pointed out in the Introduction, other mechanisms of whole-grain heating, e.g., due to inelastic grain-grain collisions, have also been suggested as a possible cause of the explosion.

Let us consider the global thermal balance for a reactive spherical grain. The steady-state temperature distribution inside the grain is almost homogeneous, and so the heating power $P_{\text {heat }}$ is approximately the product of $Q_{r} e^{-E_{a} / k_{\mathrm{B}} T}$ and the reactive (mantle) volume $4 \pi a^{2} \Delta a$. Surface cooling at temperatures above $\simeq 25 \mathrm{~K}$ is dominated by sublimation (Léger et al. 1985; Schutte \& Greenberg 1991). The resulting cooling power $P_{\text {cool }}$ is the product of the area $4 \pi a^{2}$ and the cooling rate $\Delta H_{\text {sub }}\left(2 \pi m k_{\mathrm{B}} T\right)^{-1 / 2} p_{0} e^{-\Delta H_{\text {sub }} / k_{\mathrm{B}} T}$, where $\Delta H_{\text {sub }}$ and $m$ are the sublimation enthalpy and the mass of evaporating molecules, respectively, and $p_{0}$ is the pre-factor for the saturated vapor pressure (Léger 1983; Léger et al. 1985). By substituting the heat rate $Q_{r}=E_{r} \varphi_{A} \varphi_{B} N \nu$ for reactive species $A$ and $B$, we obtain the heating-to-cooling power ratio,

$$
\frac{P_{\text {heat }}}{P_{\text {cool }}} \sim \varphi_{A} \varphi_{B} \frac{E_{r} N \Delta a \nu \sqrt{2 \pi m k_{\mathrm{B}} T}}{\Delta H_{\text {sub }} p_{0}} e^{\left(\Delta H_{\text {sub }}-E_{a}\right) / k_{\mathrm{B}} T},
$$

which must exceed unity for the temperature to increase with time. For estimates, we set $T=30 \mathrm{~K}$ which ensures that, irrespective of the poorly known emission efficiency of grains, radiative cooling is negligible (Léger et al. 1985; Schutte \& Greenberg 1991; Shen et al. 2004). By adopting $E_{a} / k_{\mathrm{B}} \simeq$ $\Delta H_{\text {sub }} / k_{\mathrm{B}} \simeq 1150 \mathrm{~K}$ for CO molecules, $\Delta a=2 \times 10^{-6} \mathrm{~cm}$ for the mantle thickness, and $p_{0} \simeq 10^{12} \mathrm{dyne}^{-2}$ for the saturated CO-vapor pressure (Léger et al. 1985), we get $P_{\text {heat }} / P_{\text {cool }} \sim 10^{-4}$ (for the reaction between $\mathrm{CO}$ and $\mathrm{OH}$ ).

We see that global grain cooling is much more efficient than heating. The temperature could only increase with time if the sublimation enthalpy $\Delta H_{\text {sub }}$ would be substantially larger than the activation energy $E_{a}$, say by several hundreds of $\mathrm{K}$. However, as was pointed out in Section 3.1, these two values are estimated to be about the same, ${ }^{6}$ and therefore it is rather unlikely that whole-grain heating could trigger the thermal explosion.

Under-critical energetic particles colliding with a grain may nevertheless stimulate reactions between radicals stored in the mantle (e.g., Reboussin et al. 2014). To estimate this effect (assuming whole-grain heating), we compare the characteristic timescales of sublimation cooling and the chemical reactions. The time to burn the characteristic fraction $\bar{\varphi}=\sqrt{\varphi_{A} \varphi_{B}}$ of the major reactive species at a given temperature is $t_{\text {chem }} \sim(\bar{\varphi} \nu)^{-1} e^{E_{a} / k_{\mathrm{B}} T}$, while the cooling time is $t_{\text {cool }} \sim E_{\text {dep }} / P_{\text {cool }}$, where $E_{\text {dep }}$ is the total energy deposited in a grain. By substituting $E_{\mathrm{dep}} \simeq 2 q_{2} a<10^{4} \mathrm{eV}$ for CR protons and otherwise using the same parameters as above (and also taking into account the crossover to radiative cooling at lower temperatures), we obtain $t_{\text {cool }} / t_{\text {chem }}<3 \times 10^{-5}$.

We conclude that the chemical reactions stimulated by whole-grain heating, due to collisions with under-critical particles, are many orders of magnitude slower than the cooling. Even though the collisions with (under-critical) CR protons are $\sim \phi_{\mathrm{Fe}}^{-1} \sim 10^{4}$ more frequent than with (over-critical) iron ions, such reactions are not expected to noticeably affect the chemical composition of the ice mantle at a timescale of the explosive disruption (although the abundances of trace species, such as complex organic molecules, may be changed, e.g., Reboussin et al. 2014). It must be stressed, however, that the above estimates completely neglect the effects of local thermal spikes generated in the mantle by CR protons, at a timescale of thermal diffusion. The question of whether the integral effect of the heterogeneous chemistry stimulated by such heating is more profound than that due to whole-grain heating requires a separate careful study.

\footnotetext{
6 Recent studies (Ghesquière et al. 2015) suggest that the diffusion energy of $\mathrm{CO}$ molecules in the bulk ice (which determines the magnitude of $E_{a}$ ) might significantly exceed the value of $\Delta H_{\text {sub }}$. If so, then the ratio $P_{\text {heat }} / P_{\text {cool }}$ would be even smaller than estimated above.
} 


\section{CONCLUSIONS}

The main result of this article is that we have identified the regime of localized spot heating for a reactive medium, and developed a rigorous theory describing the thermal evolution in this case. The problem is characterized by a single dimensionless number $\lambda$ which depends on the deposited energy and properties of the medium. The theory allows us to determine the explosion threshold and accurately describe the impulsive heating of icy mantles by energetic particles.

A collision with an over-critical energetic particle (when $\lambda$ exceeds the threshold) leads to the thermal explosion which, in turn, generates the flame front propagating in the mantle and leading to its disruption. We showed that heavy CR species, such as iron ions, are able to trigger the explosion, while the stopping power of the most abundant CR protons is insufficient for that (since the stopping power is roughly proportional to the squared atomic number of CR ions). Also, we practically ruled out other energetic species, e.g., X-rays, as possible causes of explosion due to impulsive heating.

It is important to stress that the question of how exactly the disruption occurs-whether the mantle is completely evaporated due to thermal explosion, or if a part of it is ejected off the grain in the form of tiny ice pieces-remains unclear. Thus, the possibility of the partial mechanical disruption of the mantle leads to the conclusion that the interstellar medium may contain solid nanoparticles of predominantly water ice.

Interestingly, the existence of a well-defined explosion threshold allows us to estimate also the upper limit of the abundance of radicals that may be stored in the mantle: for the assumed dust and CR properties, the product of the fractional abundances of two major radicals cannot exceed a value of $\sim 3 \times 10^{-3}$ to avoid unrealistically large desorption rates. Thus, the presented theory enables us to place constraints on astrochemical models.

When $\lambda$ is below the threshold, the deposited heat is quickly redistributed over the entire grain volume, i.e., the whole-grain heating scenario is realized. The rates of reactions between radicals frozen in the mantle exponentially depend on the temperature, and so even a slight temperature increase can dramatically accelerate the release of chemical energy in the reactive volume-for this reason, whole-grain heating has been considered so far as the prime possible cause of thermal explosion. However, we have demonstrated that an explosion is unlikely in this case, since cooling from the grain surface (due to sublimation of volatile species) turns out to be very efficient.

The non-explosive chemical processes, induced in the mantle by under-critical impulsive heating, represent another very important phenomenon which needs to be further investigated. We considered whole-grain heating due to $\mathrm{CR}$ protons, and demonstrated that in this case, the abundance of the major reactive species is not expected to noticeably change on the timescale of the explosive disruption (caused by heavy CR species). However, chemical reactions depend on the local temperature and therefore evolve much faster during short transient heating events within small volumes where undercritical particles deposit their energy. Careful analysis of such heterogeneous chemistry (as opposed to the chemistry due to whole-grain heating) and evaluation of its integral effect will be reported in a future paper.

The authors acknowledge Malcolm Walmsley for critical reading of the manuscript and helpful comments. A.V. and P.C. acknowledge support from the European Research Council (ERC, project PALs 320620).

\section{APPENDIX A RELATION BETWEEN UNSTEADY AND STEADY PROBLEMS}

Consider a reactive medium which has a characteristic size $r_{0}$ and temperature $T_{0}$ at the boundary, and assume that there is thermal equilibrium. The stability of such a steady state is determined by the Frank-Kamenetskii number (Frank-Kamenetskii 1969; Landau \& Lifshitz 1987),

$$
\lambda_{\mathrm{FK}}=\frac{Q_{r} E_{a} r_{0}^{2} e^{-E_{a} / k_{\mathrm{B}} T_{0}}}{\kappa k_{\mathrm{B}} T_{0}^{2}},
$$

which is the ratio of the timescales of thermal diffusion to chemical reaction: when $\lambda_{\mathrm{FK}}$ exceeds a certain threshold, the diffusive loss cannot compensate for a temperature increase due to the ongoing reaction and the steady state becomes unstable, i.e., a thermal explosion is triggered. The thresholds for $D=1,2$, and 3 are $\lambda_{\mathrm{FK}, \mathrm{cr}}=0.88,2$, and 3.32 , respectively (Frank-Kamenetskii 1969).

For the unsteady problem studied in this paper, the number $\lambda$ plays the role of $\lambda_{\mathrm{FK}}$. To understand their relation, let us calculate the "momentary" value of $\lambda_{\mathrm{FK}}$ for the unsteady process. The relevant scale for $T_{0}$ would be the temperature $T(0, t)$ at the center of the ignition spot, while for $r_{0}^{2}$ one should substitute the squared diffusion length $\left(q_{D} / \rho c T_{0}\right)^{2 / D}$. Then, by employing Equation (6), we obtain

$$
\lambda_{\mathrm{FK}} / \lambda \sim e^{-1 / \theta_{0}} \theta_{0}^{-2(1+1 / D)},
$$

where $\theta_{0}(t)=k_{\mathrm{B}} T(0, t) / E_{a}$. We see that $\lambda_{\mathrm{FK}} / \lambda$ is the sole function of $\theta_{0}$, and thus of $t$. It attains maximum at $\theta_{0}=2(1+1 / D)$, where $\lambda_{\mathrm{FK}} / \lambda \sim 1$, which identifies the "optimum moment" to trigger the explosion (provided $\left.\lambda>\lambda_{\text {cr }}\right)$. Physically, the optimum comes out because the size of the reactive zone is too small in the beginning (i.e., the timescale of thermal diffusion is short), while at later times the reaction becomes exponentially slow.

The derived relation allows us to obtain the dependence of $\lambda_{\mathrm{cr}}$ on the size of the ignition spot $w$. Using the relation $\theta_{0} w^{D} \sim 1$ (where $w$ is in units of $r_{*}$ ), we obtain the scaling $\lambda_{\text {cr }}(w) \sim \exp \left(w^{D}\right) w^{-2(1+D)}$, which provides an excellent fit to the curves in Figure 2 at $w \geqslant 2$.

Thus, unlike the case of a localized ignition spot, the unsteady problem for $w \gtrsim 1$ is no longer characterized by a single dimensionless number. A similar problem of the thermal explosion of large "hot spots" was studied numerically in the 1960s by Merzhanov et al. (1963) and Merzhanov (1966), who showed that for a given initial size $r_{0}$ and temperature $T_{0}$ of the spot, the explosion threshold $\lambda_{\mathrm{FK}, \mathrm{cr}}$ has a logarithmic dependence on $T_{0}$.

\section{APPENDIX B \\ FLAME FRONT}

The explosion generates the flame front propagating away from the ignition spot. At sufficiently large times, when the front coordinate $\xi$ is much larger than the front thickness, the last term on the right-hand side of Equation (4) becomes asymptotically negligible (i.e., the front curvature is no longer 
important). Then, by separating the reactive $\left(\theta>\theta_{\mathrm{tr}}\right)$ and inert $\left(\theta<\theta_{\mathrm{tr}}\right)$ zones of the front (Frank-Kamenetskii 1969; Landau \& Lifshitz 1987), we can approximately describe the temperature profile using the following equation:

$$
\begin{aligned}
& \theta>\theta_{\mathrm{tr}}: \quad \frac{\partial \theta}{\partial \tau}=\lambda+\frac{\partial^{2} \theta}{\partial \xi^{2}}, \\
& \theta<\theta_{\mathrm{tr}}: \quad \frac{\partial \theta}{\partial \tau}=\frac{\partial^{2} \theta}{\partial \xi^{2}},
\end{aligned}
$$

where $\theta_{\mathrm{tr}} \sim 1$ is the fitting parameter (to be determined from numerical solution of Equations (4) and (5)). We search the solution in the form $\theta(\xi, \tau)=\theta(s)$ with $s=\xi-u \tau$, which yields $\theta(s)=A_{1} e^{-u s}-(\lambda / u) s+A_{2} \quad$ for $\quad \theta>\theta_{\text {tr }} \quad$ and $\theta(s)=A_{3} e^{-u s}$ for $\theta<\theta_{\mathrm{tr}}$. By setting $\theta(0)=\theta_{\mathrm{tr}}$ and taking into account that in the reactive zone $\theta(s)$ cannot grow faster than linearly, we obtain $A_{1}=0$; the constants $A_{2}$ and $A_{3}$ are determined from the continuity of $\theta$ and $\partial \theta / \partial \xi$ at $s=0$. We obtain $u=\sqrt{\lambda / \theta_{\text {tr }}}$ and

$$
\begin{array}{ll}
s<0: & \theta(s)=-\sqrt{\lambda \theta_{\mathrm{tr}}} s+\theta_{\mathrm{tr}}, \\
s>0: & \theta(s)=\theta_{\mathrm{tr}} \exp \left(-\sqrt{\lambda / \theta_{\mathrm{tr}}} s\right),
\end{array}
$$

the numerical fit yields $\theta_{\text {tr }} \simeq 1.3$. In physical units, the front speed,

$$
U=\sqrt{\frac{Q_{r} \kappa}{\theta_{\mathrm{tr}}(\rho c)^{2} E_{a}}},
$$

is determined by the reactive and transport properties of the medium.

\section{APPENDIX C THERMAL EXPLOSION WHEN $C$ OR $\chi$ ARE FUNCTIONS OF $T$}

Let us consider the case when the specific heat is a function of temperature, $c(T)$, while the thermal diffusivity $\chi=\kappa / \rho c$ is first assumed to be constant. It is convenient (Landau \& Lifshitz 1987) to introduce the enthalpy $H=\rho \int c d T \equiv F(T)$, noting that $F(T)$ is a single-valued (monotonously increasing) function. Then, Equations (1) and (2) can be rewritten in the following identical form for $H$ :

$$
\begin{aligned}
\frac{\partial H}{\partial t} & =Q_{r} e^{-E_{a} / k_{\mathrm{B}} T}+\chi\left(\frac{\partial^{2} H}{\partial r^{2}}+\frac{D-1}{r} \frac{\partial H}{\partial r}\right), \\
H(r, 0) & =q_{D} \delta_{D}(r),
\end{aligned}
$$

where $T=F^{-1}(H)$ is the inverse function. We introduce the enthalpy scale, $H_{E}=F\left(E_{a}\right)$, and conclude that the problem can be reduced to the dimensionless form of Equations (4) and (5), where (apart from the Arrhenius term) $H / H_{E}$ should be substituted for $\theta$; in the Arrhenius term, $\theta$ should be replaced with $F^{-1}\left(H / H_{E}\right)$, and

$$
\lambda=\frac{Q_{r}}{\chi H_{E}}\left(\frac{q_{D}}{H_{E}}\right)^{2 / D} .
$$

Correspondingly, the speed of the flame front is given by

$$
U=\sqrt{\frac{Q_{r} \chi}{\theta_{\mathrm{tr}} H_{E}}} .
$$

For example, for $c \propto T^{\alpha}$ with the exponent $\alpha \geqslant 0$, we get $H / H_{E}=\theta^{1+\alpha}$, where $H_{E}=\rho c\left(E_{a}\right) E_{a} /(1+\alpha)$; for a constant specific heat $(\alpha=0)$, Equations (9) and (10) are reduced to Equations (6) and (8), respectively. Figure 3 shows that the explosion threshold decreases dramatically with $\alpha$.

Also, we analyzed the effect of the temperature-dependent thermal diffusivity $\chi$. In this case, the diffusion term in the heat Equation becomes nonlinear. From the numerical solution with $\chi \propto T^{\beta}$, we obtained the dependencies $\lambda_{\text {cr }}(-\beta)$ that are qualitatively similar to $\lambda_{\text {cr }}(\alpha)$ shown in Figure 3 (i.e., $\lambda_{\text {cr }}$ monotonically increases with $\beta$ ). However, the relative variation of $\lambda_{\text {cr }}$ with $\beta$ turns out to be several times smaller than with $\alpha$, i.e., the effect of $\chi(T)$ on the explosion threshold is substantially weaker than that of $c(T)$.

\section{REFERENCES}

Andersson, O., \& Suga, H. 2002, PhRvB, 65, 140201

Andersson, S., \& van Dishoeck, E. F. 2008, A\&A, 491, 907

Bacmann, A., Taquet, V., Faure, A., Kahane, C., \& Ceccarelli, C. 2012, A\&A, 541, L12

Bizzocchi, L., Caselli, P., Spezzano, S., \& Leonardo, E. 2014, A\&A, 569, A27 Bringa, E. M., \& Johnson, R. E. 2004, ApJ, 603, 159

Brünken, S., Sipilä, O., Chambers, E. T., et al. 2014, Natur, 516, 219

Caselli, P., Benson, P. J., Myers, P. C., \& Tafalla, M. 2002, ApJ, 572, 238

Caselli, P., \& Ceccarelli, C. 2012, A\&ARv, 20, 56

Caselli, P., Keto, E., Bergin, E. A., et al. 2012, ApJL, 759, L37

Cecchi-Pestellini, C., Duley, W. W., \& Williams, D. A. 2012, ApJ, 755, 119

Cernicharo, J., Marcelino, N., Roueff, E., et al. 2012, ApJL, 759, L43

Chang, Q., \& Herbst, E. 2014, ApJ, 787, 135

Cruz-Diaz, G. A., Muñoz Caro, G. M., Chen, Y.-J., \& Yih, T.-S. 2014, A\&A, 562, A119

Cuppen, H. M., Morata, O., \& Herbst, E. 2006, MNRAS, 367, 1757

d'Hendecourt, L. B., Allamandola, L. J., Baas, F., \& Greenberg, J. M. 1982, A\&A, 109, L12

Duley, W. W., \& Williams, D. A. 1993, MNRAS, 260, 37

Frank-Kamenetskii, D. A. 1969, Diffusion and Heat Transfer in Chemical Kinetics (New York: Plenum)

Garrod, R. T. 2013a, ApJ, 765, 60

Garrod, R. T. 2013b, ApJ, 778, 158

Garrod, R. T., Wakelam, V., \& Herbst, E. 2007, A\&A, 467, 1103

Ghesquière, P., Mineva, T., Talbi, D., et al. 2015, PCCP, submitted

Gibb, E. L., Whittet, D. C. B., Boogert, A. C. A., \& Tielens, A. G. G. M. 2004, ApJS, 151, 35

Hartquist, T. W., \& Williams, D. A. 1990, MNRAS, 247, 343

Hasegawa, T. I., \& Herbst, E. 1993, MNRAS, 261, 83

Hasegawa, T. I., Herbst, E., \& Leung, C. M. 1992, ApJS, 82, 167

Herbst, E., \& Cuppen, H. M. 2006, PNAS, 103, 12257

Landau, L. D., \& Lifshitz, E. M. 1987, Fluid Mechanics (Oxford: Pergamon)

Léger, A. 1983, A\&A, 123, 271

Léger, A., Jura, M., \& Omont, A. 1985, A\&A, 144, 147

Merzhanov, A. G. 1966, CoFl, 10, 341

Merzhanov, A. G., Barzykin, V. V., \& Gontkovskaya, V. T. 1963, DoSSR, 148,380

Myers, P. C., Fuller, G. A., Mathieu, R. D., et al. 1987, ApJ, 319, 340

Öberg, K. I., Bottinelli, S., Jørgensen, J. K., \& van Dishoeck, E. F. 2010, ApJ, 716,825

Padovani, M., Galli, D., \& Glassgold, A. E. 2009, A\&A, 501, 619

Petrovic, J. J. 2003, JMatS, 38, 1

Rawlings, J. M. C., Williams, D. A., Viti, S., Cecchi-Pestellini, C., \& Duley, W. W. 2013, MNRAS, 430, 264

Reboussin, L., Wakelam, V., Guilloteau, S., \& Hersant, F. 2014, MNRAS, 440, 3557

Roberts, J. F., Rawlings, J. M. C., Viti, S., \& Williams, D. A. 2007, MNRAS, 382, 733

Ruffle, D. P., \& Herbst, E. 2000, MNRAS, 319, 837

Schutte, W. A., \& Greenberg, J. M. 1991, A\&A, 244, 190 
Shalabiea, O. M., \& Greenberg, J. M. 1994, A\&A, 290, 266

Shen, C. J., Greenberg, J. M., Schutte, W. A., \& van Dishoeck, E. F. 2004, A\&A, 415, 203

Tafalla, M., Myers, P. C., Caselli, P., \& Walmsley, C. M. 2004, A\&A, 416, 191

Tafalla, M., Myers, P. C., Caselli, P., Walmsley, C. M., \& Comito, C. 2002, ApJ, 569, 815
Taquet, V., Ceccarelli, C., \& Kahane, C. 2012, A\&A, 538, A42

Vasyunin, A. I., \& Herbst, E. 2013a, ApJ, 762, 86

Vasyunin, A. I., \& Herbst, E. 2013b, ApJ, 769, 34

Vogt, C., Laihem, K., \& Wiebusch, C. 2008, ASAJ, 124, 3613

Zeller, R. C., \& Pohl, R. O. 1971, PhRvB, 4, 2029

Ziegler, J. F. 1980, Stopping Cross-Sections for Energetic Ions in All Elements, Vol. 5 (New York: Pergamon) 